\title{
ON THE FINAL SET OF A REAL ENTIRE FUNCTION OF EXPONENTIAL TYPE
}

\author{
MURALI RAO AND LI-CHIEN SHEN
}

\begin{abstract}
In this paper we have constructed a real entire function of exponential type which is bounded on the real axis such that the entire real axis belongs to its final set.
\end{abstract}

1. Introduction. Following Pólya, the final set of an entire function $f$ is the set of accumulation points of the set

$$
\left\{z ; f^{(n)}(z)=0 \text { for some } n \geq 0\right\} \text {. }
$$

We say that an entire function $f(z)$ is real if it takes real values for real $z$, or what is the same, the coefficients of its Maclaurin series are all real.

In his well-known address presented to the American Mathematical Society, April 3, 1942, Pólya asserted that [1, p. 181]:

"For certain entire functions of order 1, as, for example, for

$$
f(z)=\sin z
$$

the differentiation does not change essentially the density of the distribution of the zeros."

In view of the above general assertion of Pólya, the following conjecture seems plausible.

HYPOTHETICAL THEOREM. Let $f$ be a real entire function of order one, exponential type $\rho$, and bounded on the real axis. Then the final set of $f$ is an equally spaced infinite set on the real axis.

(The spacing between any two adjacent points is $\pi / 2 \rho$.)

Recently, C. Prather [3] published a theorem which confirms the Hypothetical Theorem. However, his proof contains an error. To be precise, we point out where this error occurs. On p. 367, he stated in the last paragraph

"For case (b) where $\tau=\lambda$, we argue as follows. When strict inequality holds in (3.4), for all $m=1,2,3, \ldots$, the preceding argument applies to establish the result."

The above statement is not a valid one; since to establish the crucial (3.8) of [3, p. 367], it is absolutely essential that $\tau<\lambda$. In fact, the error is serious; since, in this note, we have constructed a counterexample which disproves the above Hypothetical Theorem.

The main purpose of this paper is to prove the following theorem.

Received by the editors September 19, 1985 and, in revised form, February 3, 1986.

1980 Mathematics Subject Classification (1985 Revision). Primary 30A66; Secondary 30A66.

Key words and phrases. Real entire function, exponential type, final set. 
THEOREM 1. There exists a real entire function of exponential type and bounded on the real axis such that the whole real axis belongs to its final set.

It must be pointed out here that Theorem 1 does not contradict Pólya's assertion, for Pólya only stated that "the density" of the distribution of the zeros of $f^{(n)}$ does not change essentially. The reader will notice that in our constructed function $F$, the spacing of the zeros of a subsequence of $\left\{F^{(n)}\right\}$ does converge to $\pi / 2$, but the zeros are "drifting" around the real axis to fill up the entire real axis.

The function $F$ to be constructed is of the form

$$
F(z)=\int_{0}^{1} \cos z t d \lambda(t)+\int_{0}^{1} \sin z t d t
$$

where the finite positive measure $\lambda$ is to be constructed in $\S 2$. All the needed lemmas are stated in $\S 3$ and Theorem 1 is proved in $\S 4$.

2. Construction of the measure $\lambda$. Let $\left\{b_{j}\right\}$ be a countable set dense in $[0, \infty)$. Let the sequence $\left\{n_{j}\right\}_{j=1}^{\infty}$ of positive even integers be chosen inductively as follows: $n_{1}=2$. For each $n_{j}$, choose $n_{j+1}$ sufficiently large so that:

(a) $n_{j+1}>n_{j}$

(b) $n_{j+1}>n_{j} b_{j+1}(j+1)^{2}$, and

(c) $n\left(2^{-1 / n_{j}}\right)^{n}<1 / j$ for all $n \geq n_{j+1}$.

Then, for each $j$, define $t_{j}=2^{-1 / n_{j}}$. Then (c) gives

(d) $n t_{j}^{n}<1 / j$ for $n \geq n_{j+1}$.

We also note that

(e) $t_{j}^{n_{j}}=1 / 2$.

We now define the finite positive measure $\lambda$ on all Borel subsets of $[0,1]$ as follows.

$$
\lambda\left(\left\{t_{j}\right\}\right)=b_{j} / n_{j} \quad \text { and } \quad \lambda\left([0,1]-\left\{t_{j}: j=1,2, \ldots\right\}\right)=0 .
$$

Clearly, from (b), $\lambda$ is a finite measure. Moreover, from (2.1),

$$
\int_{0}^{1} x^{n} d \lambda(x)=\sum_{j=1}^{\infty} \frac{b_{j}}{n_{j}} t_{j}^{n}
$$

We now prove the following crucial property of $\lambda$.

LEMMA 1 .

$$
\left|n_{i} \int_{0}^{1} x^{n_{i}} d \lambda-\frac{b_{i}}{2}\right| \rightarrow 0 \quad \text { as } i \rightarrow \infty
$$

Proof. Write the right-hand side of (2.2) as the sum of $I_{1}, I_{2}$, and $I_{3}$, where, for $i \geq 2$,

$$
I_{1}=\frac{b_{i}}{2 n_{i}}, \quad I_{2}=\sum_{j=1}^{i-1} \frac{b_{j}}{n_{j}} t_{j}^{n_{i}}, \quad I_{3}=\sum_{j=i+1}^{\infty} \frac{b_{j}}{n_{j}} t_{j}^{n_{i}} .
$$

Using (c) and the fact that $t_{i-1}>t_{j}$ for $j=1,2, \ldots, i-2$, we have

$$
n_{i} I_{2}=\sum_{j=1}^{i-1} \frac{b_{j} n_{i} t_{j}^{n_{i}}}{n_{j}}<\sum_{j=1}^{i-1} \frac{b_{j} n_{i} t_{i-1}^{n_{i}}}{n_{j}}<\sum_{j=1}^{i-1} \frac{b_{j}}{n_{j}} \frac{1}{i-1} .
$$


From (b), we see that the last sum is bounded by

which tends to 0 as $i \rightarrow \infty$.

$$
\frac{1}{i-1}\left(b_{1}+\sum_{j=2}^{\infty} \frac{1}{j^{2}}\right)
$$

Since $t_{j}<1$, (b) implies that

$$
n_{i} I_{3}<\sum_{j=i+1}^{\infty} \frac{n_{i} b_{j}}{n_{j}}<\sum_{j=i+1}^{\infty} \frac{1}{j^{2}} \rightarrow 0
$$

as $i \rightarrow \infty$.

3. Some basic lemmas. Let $\nu$ be a positive Borel measure with support $[a, 1]$ for some $0 \leq a \leq 1$. Define

$$
a_{n}=\int_{0}^{1} t^{n} d \nu, \quad n=0,1,2, \ldots
$$

LEMMA 2.

$$
\frac{1}{a_{n}} \int_{0}^{1} t^{n}(1-t) d \nu \rightarrow 0 \quad \text { as } n \rightarrow \infty .
$$

PROOF. For any $\varepsilon>0$, choose $u_{0}$ and $u_{1}$ such that

$$
a<u_{0}<u_{1}<1 \text { and } 1-u_{0}<\varepsilon / 2 \text {. }
$$

Clearly, from (3.1),

$$
\begin{aligned}
\int_{0}^{1} t^{n}(1-t) d \nu & =\int_{0}^{u_{0}} t^{n}(1-t) d \nu+\int_{u_{0}}^{1} t^{n}(1-t) d \nu \\
& <a_{0} u_{0}^{n}+\varepsilon a_{n} / 2 \\
a_{n} & >\int_{u_{1}}^{1} t^{n} d \nu>u_{1}^{n} m
\end{aligned}
$$

where $m=\int_{u_{1}}^{1} d \nu(>0)$.

Now we choose $N$ so that

$$
\left(a_{0} / m\right)\left(u_{0} / u_{1}\right)^{n}<\varepsilon / 2 \text { for } n \geq N .
$$

From (3.2), (3.3), and (3.4), we have

$$
\begin{aligned}
\frac{1}{a_{n}} \int_{0}^{1} t^{n}(1-t) d \nu & <\frac{1}{a_{n}}\left(a_{0} u_{0}^{n}+a_{n} \varepsilon / 2\right) \\
& <\left(a_{0} / m\right)\left(u_{0} / u_{1}\right)^{n}+\varepsilon / 2 \\
& <\varepsilon,
\end{aligned}
$$

for all $n \geq N$. This completes the proof.

LEMMA 3. Let $f(t)$ be either $\cos t$ or $\sin t$. Then

$$
\frac{1}{a_{n}} \int_{0}^{1} t^{n} f(t z) d \nu \rightarrow f(z)
$$

uniformly as $n \rightarrow \infty$ for $z$ in a compact set.

PROOF. This lemma follows immediately from Lemma 2 and the fact that $f(z t)=f(z)+O(1-t)$ for $z$ in a compact set and $t$ in $[0,1]$. 


\section{Proof of Theorem 1. Define}

$$
F(z)=\int_{0}^{1} \cos z t d \lambda+\int_{0}^{1} \sin z t d t
$$

where the measure $\lambda$ is the one that we constructed in $\S 2$. Then $F$ is a real entire function of exponential type (in fact, its type is equal to 1 ) and $F$ is bounded on the real axis.

Since differentiation under the integral is justified, we clearly have

$$
F^{(2 n)}(z)=(-1)^{n}\left\{\int_{0}^{1} t^{2 n} \cos z t d \lambda+\int_{0}^{1} t^{2 n} \sin z t d t\right\} .
$$

Hence, using the notation of Lemma 2 with $\nu=\lambda$,

$$
\begin{aligned}
(-1)^{n}(2 n+1) F^{(2 n)}(z)= & (2 n+1) a_{2 n} \frac{1}{a_{2 n}} \int_{0}^{1} t^{2 n} \cos z t d \lambda \\
& +(2 n+1) \int_{0}^{1} t^{2 n} \sin z t d t
\end{aligned}
$$

From Lemma 1 , we see that for any $\tau$ in $[0, \infty)$ we can select a subsequence $\left\{k_{j}\right\}$ from the original sequence $\left\{n_{j}\right\}$ such that

$$
\left(k_{j}+1\right) a_{k_{j}} \rightarrow \tau \text { as } j \rightarrow \infty .
$$

We remind the reader that the $k_{j}$ are even.

By application of Lemma 3 to $\lambda$ and to Lebesgue measure and by using (4.1) and (4.2), we obtain

$$
(-1)^{k_{j} / 2}\left(k_{j}+1\right) F^{\left(k_{j}\right)}(z) \rightarrow \tau \cos z+\sin z=\left(\tau^{2}+1\right)^{1 / 2} \sin (z+\theta(\tau))
$$

as $j \rightarrow \infty$, uniformly for $z$ in a compact set; where

$$
\sin \theta(\tau)=\tau /\left(\tau^{2}+1\right)^{1 / 2} \text { and } 0 \leq \theta(\tau)<\pi / 2
$$

Similarly, we have

$$
(-1)^{k_{j} / 2}\left(k_{j}+1\right) F^{\left(k_{j}+1\right)}(z) \rightarrow\left(\tau^{2}+1\right)^{1 / 2} \cos (z+\theta(\tau)) .
$$

By Hurwitz's theorem [2, p. 143], (4.3) and (4.5) imply that each zero of $\sin (z+\theta(\tau))$ or $\cos (z+\theta(\tau))$ is the limit of zeros of $F^{\left(k_{j}\right)}$ or $F^{\left(k_{j}+1\right)}$ and therefore belongs to the final set of $F$. Hence all the points $\{n \pi / 2-\theta(\tau)\}_{n=-\infty}^{\infty}$ lie in the final set of $F$. Since $\tau$ can be any value in $[0, \infty)$, we see that (4.4) implies that

$$
\{\theta(\tau): 0 \leq \tau<\infty\}=[0, \pi / 2) .
$$

It is now clear that the whole real axis is contained in the final set of $F$. This ends the proof of Theorem 1.

ACKNOWLEDGMENT. The authors would like to thank the referee for many helpful comments and suggestions which have made this paper easier to read. 


\section{REFERENCES}

1. G. Pólya, On the zeros of an entire function and its analytic character. Bull. Amer. Math. Soc. 49 (1943), 178-191.

2. G. Pólya and G. Szegö, Problems and theorems in analysis. I, English translation, SpringerVerlag, New York-Heidelberg-Berlin, 1972.

3. C. Prather, Final set for operators on real entire functions of order one, normal type, Proc. Amer. Math. Soc. 90 (1984), 363-369.

Department of MAThematics, University of Florida, Gainesville, Florida 32611 